\title{
Erratum to: TP53 and p53 statuses and their clinical impact in diffuse low grade gliomas
}

\author{
Emeline Gillet • Agusti Alentorn • Brahima Doukouré • Emeline Mundwiller • \\ Hinke F. van Thuijl · Jaap C. Reijneveld · José Alfonso Meza Medina • \\ Amélie Liou • Yannick Marie · Karima Mokhtari • Khê Hoang-Xuan • \\ Marc Sanson · Jean-Yves Delattre $\cdot$ Ahmed Idbaih
}

Published online: 5 July 2014

(C) Springer Science+Business Media New York 2014

\section{Erratum to: J Neurooncol (2014) 118:131-139 \\ DOI 10.1007/s11060-014-1407-4}

The fifth author's name was misspelled in the original publication. It is correct in this erratum.

The online version of the original article can be found under doi:10.1007/s11060-014-1407-4.

E. Gillet · A. Alentorn - E. Mundwiller · Y. Marie .

K. Mokhtari - K. Hoang-Xuan - M. Sanson - J.-Y. Delattre ·

A. Idbaih

Centre de Recherche de l'Institut du Cerveau et de la Moelle

Epinière (CRICM), Université Pierre et Marie Curie-Paris 6, UMRS 975, Paris, France

E. Gillet · A. Alentorn · E. Mundwiller · Y. Marie ·

K. Mokhtari - K. Hoang-Xuan - M. Sanson - J.-Y. Delattre ·

A. Idbaih

Inserm U 975, Paris, France

E. Gillet · A. Alentorn · E. Mundwiller · Y. Marie ·

K. Mokhtari - K. Hoang-Xuan - M. Sanson - J.-Y. Delattre .

A. Idbaih

CNRS, UMR 7225, Paris, France

E. Gillet · A. Alentorn $(\varangle) \cdot$ J. A. M. Medina

K. Hoang-Xuan · M. Sanson · J.-Y. Delattre · A. Idbaih AP-HP, Groupe Hospitalier Pitié-Salpêtrière, Service de Neurologie 2-Mazarin, 47-83 Boulevard de l'Hôpital, 75013 Paris, France

e-mail: agusti.alentorn@gmail.com
E. Gillet · A. Liou

AP-HP, Groupe Hospitalier Pitié-Salpêtrière, Service de Pharmacie, Paris, France

B. Doukouré · K. Mokhtari

AP-HP, Groupe Hospitalier Pitié-Salpêtrière, Service de

Neuropathologie Raymond Escourolle, Paris, France

H. F. van Thuijl · J. C. Reijneveld

Department of Neurology, VU University Medical Center, Amsterdam, The Netherlands 